\title{
Automatic Segmentation of Myocardial Infarction in Rats Subjected to Regional Ischemia
}

\author{
R Jakubicek $^{1}$, J Chmelik ${ }^{1}$, J Neckar ${ }^{2}$, R Kolar ${ }^{1}$ \\ ${ }^{1}$ Department of Biomedical Engineering, Faculty of Electrical Engineering and Communication, \\ Brno University of Technology, Brno, Czech Republic \\ ${ }^{2}$ Institute of Physiology, Czech Academy of Sciences, Prague, Czech Republic
}

\begin{abstract}
The experimental and preclinical studies of ischemia and reperfusion on animal models usually evaluate the sizes of area at risk (AR) of infarction and infarct area (IA) as fundamental parameters. The authors usually don't provide any detailed information about the image processing of their data, though the IA or AR segmentation is often challenging and prone to be expert-depending. Here, we describe a new approach for automatic IA and AR segmentation based on combination of Random Forest classifier and two-step pixel-wise k-means classification of image pixels. The evaluation has been performed on the set of 16 images from 8 rat hearts. We compared sizes of normal perfused tissues, viable area and IA (normalized to percentage of total area) obtained by our method with manually segmentation by biologist. We achieved mean absolute error of $2.59 \%$ with mean standard deviation of $1.61 \%$.
\end{abstract}

\section{Introduction}

Ischemic heart diseases (IHD) are the leading cause of morbidity, mortality, and disability in the worldwide and remain one of the greatest challenges in biomedical research. Although considerable progress in a management of IHD therapies has been made, novel therapeutic strategies are required.

In the experimental and preclinical studies, the determination of the extent of myocardial ischemic injury is an essential prerequisite to the study of ischemia and reperfusion. Indeed, determination of area at risk (AR) of infarction and infarct area (IS) is fundamental (and gold standard) for the evaluation of the degree of ischemic injury and potency of cardioprotective interventions [1]. Usually, the IS is normalized to the AR or left ventricle (LV) size (IS/AR and IS/LV, respectively) and AR is normalized to the LV (AR/LV).

Although different authors used different staining methods in their protocols, the assessment of infarct size is de- scribed relatively vaguely. Few papers mention different software but without any detail description of image processing methodology; e.g. Tang et al. [2] and Yan et al. [3] used popular free ImageJ software, Zhou et al. [4] used ImagePro 6.0 Plus or Yuan et al. [5] used Motic Med 6.0. The authors usually don't provide any detailed information about the image processing in their software. As the IS or AR segmentation is often challenging, the subjective segmentation might provide biased, expert-depending results.

In this paper we describe an novel approach for automatic and semi-automatic segmentation of infarct area (IA), tissue that survived ischemia (viable area VA) and normally perfused LV tissue (NP) in order to provide detailed description of the image processing methods. For the possibility of objective comparison of automatic and manual approach, the resulting area sizes (IA, VA and NP) have been normalized to the total area (LV).

\section{Methods}

In this section we briefly explain a procedure of ischemia/reperfusion (I/R) of the experimental subjects, followed by description of tissue staining process, the preparation of histological slices of myocardium and image acquisition. Next, we present a fully-automatic method that autonomously solve both of the segmentation problems (slices and tissues). If the results of automatically segmented tissues are not sufficient, the manual editing tools could be used. All proposed image processing methods were implemented in Matlab, and they are publicly available as a freeware software (www.mathworks.com/matlabcentral/fileexchange/66902cardiac-ischemia). A basic pipeline of the algorithm is outlined in Figure 1.

\subsection{Data}

Rats were subjected to acute ischemia/reperfusion as described previously in [6]. Briefly, anesthetized (sodium pentobarbital, $60 \mathrm{mg} / \mathrm{kg}$ i.p.) animals were intubated with 
a cannula connected to a rodent ventilator and ventilated with room air at $68-70$ strokes/min (tidal volume of 1.2 $\mathrm{ml} / 100 \mathrm{~g}$ body weight). The rectal temperature was maintained between 36.5 and $37.5^{\circ} \mathrm{C}$ by a heated table throughout the experiment.

Left thoracotomy was performed and a silk braided suture $5 / 0$ was placed around the left anterior descending (LAD) coronary artery about $12 \mathrm{~mm}$ distal to its origin. After 15-min stabilization, regional myocardial ischemia was induced by the tightening of the suture threaded through a polyethylene tube. After 20-min occlusion period, the ligature was released and chest was closed, air was exhausted from thorax and spontaneously breathing animals were maintained in deep anesthesia for $3 \mathrm{hrs}$.

After $3 \mathrm{hrs}$ of reperfusion, the hearts were excised and washed with $20 \mathrm{ml}$ saline through the cannulated aorta. The LAD coronary artery was then re-occluded and the ischemic zone was delineated by staining the non-ischemic tissue with $5 \%$ potassium permanganate dissolved in water $(2 \mathrm{ml}$ through the aorta). After washing out the solution thoroughly with saline and releasing the occlusion, the hearts were frozen and cut perpendicularly to the long axis of the ventricle into slices approx. $1 \mathrm{~mm}$ thick and incubate 30 min with 1\% 2,3,5-triphenyltetrazolium chloride dissolved in $0.1 \mathrm{~mol} / \mathrm{l}$ phosphate buffer $(\mathrm{pH} 7.4)$ to stain surviving myocardium of the area at risk. Next, slices were stored in $10 \%$ neutral formaldehyde solution.

Four days after the infarct size staining, the right ventricular free wall was separated and both sides of the slices were photographed on Petri dish by digital camera Nikon D60 with macro objective Micro Nikkor $60 \mathrm{~mm}$. The final image is illustrated as Input Image in Figure 1.

\subsection{Fully-automatic segmentation}

The first step consists of separation of background and histological slices (one image contains from 5 to 8 slices) by a pixel-wise Random Forests (RF) classification [7]. A vector of nine features for each pixel is used as an input of the RF, where three of them are normalized RGB image components of the original image; six additional features are also formed by RGB components, but they are obtained from two blurred versions of the processed image. The blurring is done by a Gaussian kernel with $\sigma=2$ and $\sigma=7$, respectively. The segmented binary masks are then refined by a sequence of standard morphological operations (median filter, filling holes and area filter). These segmented slices are used for creating the individual slice sub-images (each sub-image contains one segmented histological slice) for the next processing step (see Segmented Slices in Figure 1).

A learning of the RF classifier was performed on a learning set of six images with a manually labelled background and slice areas.

The second step of the proposed algorithm deals with the segmentation of the tissues of interest (IA, VA, NP). For this purpose a two-step pixel-wise k-means classification [8] is used, where the knowledge about colour properties of stained tissues are exploited. Firstly, the AR (IA+VA) tissue is separated from the normally perfused tissue - here, these areas contain shades of yellow/light brown, dark brown and black colours. On the other hand, they also contain colours from light pink, via red to white. Consequently, a main difference between the NP and AR tissue is noticeable in values of a red colour in RGB space; only the red image component is used in this case. Secondly, the IA tissue is segmented from the previously obtained AR tissue. To distinguish white/pink shades of the IA tissue from the red shades of the VA, the green and blue image components are used; the red component is saturated for both of tissues, and therefore does not provide any useful information. The segmented tissue masks are finally refined by a sequence of standard morphological operations (filling holes and area filter). Final segmentation is depicted as Segmented Tissues in Figure 1.

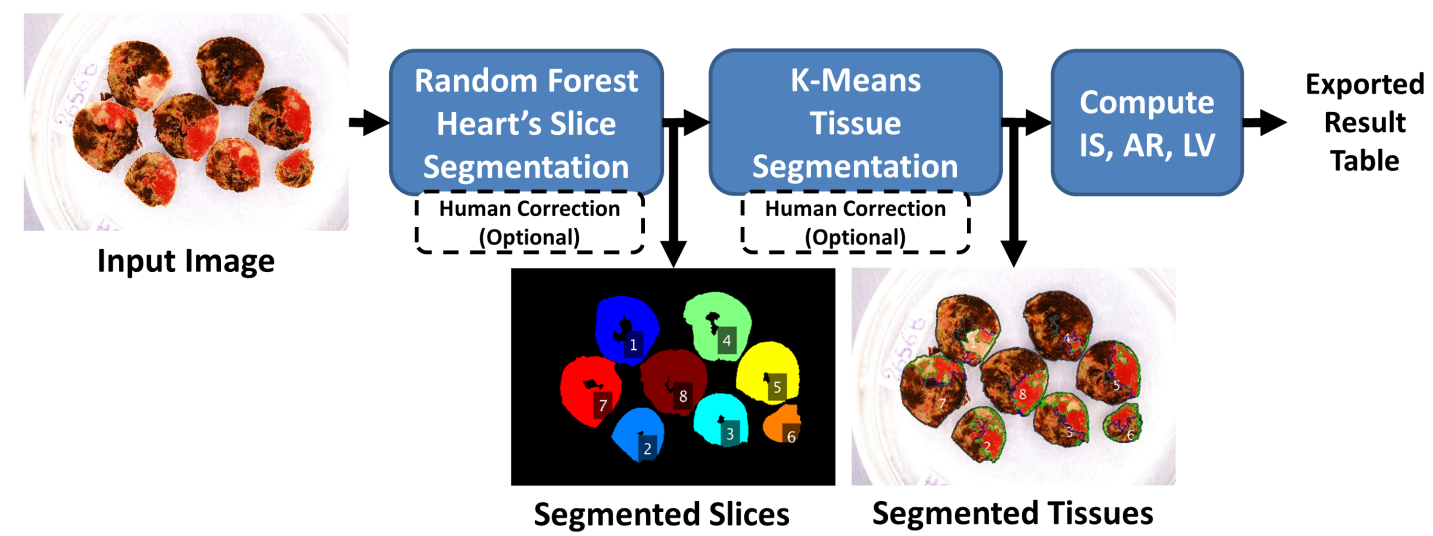

Figure 1. Basic block diagram of the fully-automatic segmentation process. 
In order to speed-up the automatic segmentation algorithm, the image processing steps are performed on the downsampled images, and resulting segments are backwards upsampled to the original size.

Finally, the segmented tissues (IA, VA, NP) are converted to biologically interesting tissues $(I S=I A, A R=$ $I A+V A, L V=I A+V A+N P)$, and usually used parameters $(I S / A R, I S / L V, A R / L V)$ are then computed. Thus obtained objective results, together with segmented image, are exported.

\subsection{Human correction tools}

In the cases, where the results of the automatic segmentation steps are not satisfactory, the user can use semiautomatic tools to edit segmented tissues. These tools could be used in any step of segmentation (slices/tissues). A common tool is freehand pen that enables simple sketching of any closed shape, which could be added or removed from the actually modified segment.

More sophisticated tool (Figure 2) is based on the division of the image to several parts with homogeneous colours, where each part can be added or removed from the modified segment by simple mouse-click. The division is performed by a watershed [9] algorithm applied on a gradient image. A size of the homogeneous parts could be controlled by a single steerable threshold, which combines several parameters: a size of the median filter, a number of quantization levels, and a level of used h-minima transform [10]. Higher threshold means larger areas.

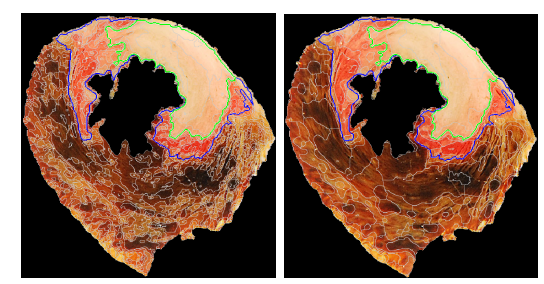

Figure 2. Example of manual correction by homogeneity tool. Blue contour defines AR tissue, green contour represents IA tissue. White contours divide image into homogeneous parts. Left image shows example with lower threshold value than right image.

\section{Experiments and results}

The dataset of 16 images from 8 rat's hearts has been used for evaluation. These images were manually segmented using a computerized planimetric method developed at Institute of Physiology (Czech Academy of Sciences, Prague, Czech Republic) few years ago.

Although, in the first step of our algorithm, we segment the individual heart slices, there is not an option to eval- uate its efficiency, because the old method used for the manual annotation does not provide segmented tissue areas, but only the their sizes. For this reason, we present the evaluation of the final segmentation of individual tissues and compare it with the manual approach. Nevertheless, these eventual segmentation errors from the first step influence the accuracy assessment of the tissue segmentation (see bellow).

For the efficiency evaluation, the mean absolute errors (mAE) and standard deviation of absolute errors (sAE) has been used. Each of tissue areas is normalized by total size of heart slice and presented in percentage. Then, the error for each type of tissue is defined as the difference between these relative area sizes obtained by old manual method and proposed automatic approach. The achieved results of the automatic system (without a human editing) are summarized in Table 1 and illustrated in Figure 3.

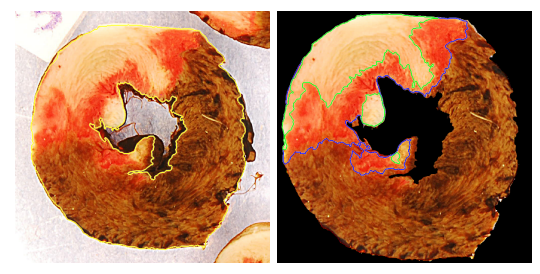

Figure 3. Examples of the segmentation results: first column illustrates the segmentation of individual histological slice (yellow contour). In the second column examples of the segmented areas of interest are shown. Blue contour defines AR tissue, green contour represents IA tissue.

Table 1. Resulting mean absolute errors (mAE) and standard deviation of absolute errors (sAE) between manual and fully automatic approach. The areas of individual tissues are related to overall area of heart slice.

\begin{tabular}{lccc}
\hline \hline Area & $\begin{array}{c}\text { Mean tissue } \\
\text { ratio [\%] }\end{array}$ & mAE [\%] & sAE [\%] \\
\hline Normally perfused (NP) & 59.71 & 2.71 & 1.78 \\
Viable area (VA) & 21.17 & 1.24 & 1.30 \\
Infarct area (IA) & 19.12 & 3.81 & 1.74 \\
\hline \hline
\end{tabular}

As shown in Table 1, the most accurate segmentation result with mean error of $1.24 \%$ is observed in VA, whereas the highest mean error $(3.81 \%)$ is achieved for IA. Because the segment of this type of tissues are naturally less compact shaped (as can be seen in Figure 4) and moreover, there are more fuzzy boundaries between IA and VA, the higher error of IA can be expected.

One of the possible problematic cases is the visibility of external and internal walls of myocardium which should be classified into background. It is due to the nonzeros thickness of slices and because the heart has a conical shape. As can be seen in Figure 4 (third image from the left), automatic algorithm provides segmentation of the LV (yellow 

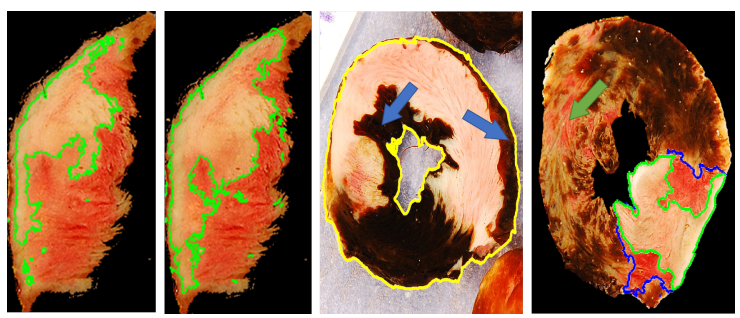

Figure 4. Examples of the problematic cases in AR and LV. First two images from the left illustrate two segmentation possibilities caused by the fuzziness of the borders between the IA (indicated by green contour) and VA in AR. Third image illustrates a problematic presence of walls of myocardium that are falsely classified as part of tissues of interest (blue arrows). A dislocation of VA tissue caused by IA transparency is shown in fourth image (green arrow).

coloured contour) including these unwanted areas (blue arrows), which then negatively affects the segmentation accuracy. Another problem is a dislocation of VA into normal perfused tissues, which is caused by the transparency of IA tissue from deeper layers (green arrow). The classification of this areas is very vague and influences resulting accuracy of the segmentation. Because of these problematic cases, the option of the user editing of the segmented areas was added into our pipeline of the automatic system.

Nevertheless, it should be emphasized that all received results for the automatic system are distinctly under of 5\%, which is error that corresponds to an average inter-observer error, heuristically estimated by the biologists at Institute of Physiology.

Also the standard deviations, which do not exceed the $2 \%$ for all tissues, prove that proposed automatic system is applicable in the experimental and preclinical studies dealing with assessment of regional ischemia.

The considerable yield of our automatic system is large decrease of the time requirements on the evaluation of the degree of ischemic injury. The old manual planimetric annotation takes about 2-3 hours per one heart, whereas our algorithm provides results in approximately 2 minutes. In the case of using a manual editing of the automatically obtained segmentation result, the overall computational time is naturally higher by 20-30 minutes per one heart (in average by 2 minutes per histological slice), nevertheless it assumes an improvement in segmentation accuracy.

\section{Conclusion}

This paper describes a two-stage segmentation technique for processing of histological slices of stained heart based on Random Forest classification and k-means clustering. The main information for segmentation is included in the colour of the stained tissues. Therefore, we used vec- tor of RGB based features with few modifications. The experimental results confirm that proposed approach is efficient for segmentation of three types of tissues, used for estimation of common parameters (IS, AR, LV) in ischemia studies. The implementation is publicly available as a Matlab source code for research. The application is completed by additional options enabling the manual correction of segmentation, which increase its versatility.

\section{Acknowledgements}

This work has been supported by the Czech Science Foundation, grant number 18-03207S.

\section{References}

[1] Ostadal B, Kolar F. Cardiac ischemia: From injury to protection. Springer, 1999.

[2] Tang XL, Rokosh G, Sanganalmath SK, Yuan F, Sato H, Mu J, Dai S, Li C, Chen N, Peng Y, et al. Intracoronary administration of cardiac progenitor cells alleviates left ventricular dysfunction in rats with a 30-day-old infarction. Circulation 2010;121(2):293-305.

[3] Yan X, Shichita T, Katsumata Y, Matsuhashi T, Ito H, Ito K, Anzai A, Endo J, Tamura Y, Kimura K, et al. Deleterious effect of the IL-23/IL-17A axis and $\gamma \delta$ T cells on left ventricular remodeling after myocardial infarction. Journal of the American Heart Association 2012;1(5):e004408.

[4] Zhou Xl, Wan L, Xu Qr, Zhao Y, Liu Jc. Notch signaling activation contributes to cardioprotection provided by ischemic preconditioning and postconditioning. Journal of translational medicine 2013;11(1):251.

[5] Yuan F, Zhang L, Li YQ, Teng X, Tian SY, Wang XR, Zhang Y. Chronic intermittent hypobaric hypoxia improves cardiac function through inhibition of endoplasmic reticulum stress. Scientific reports 2017;7(1):7922.

[6] Neckar J, Papousek F, Novakova O, Ostadal B, Kolar F. Cardioprotective effects of chronic hypoxia and ischaemic preconditioning are not additive. Basic research in cardiology 2002;97(2):161-167.

[7] Breiman L. Random forests. Machine learning 2001; 45(1):5-32.

[8] Arthur D, Vassilvitskii S. k-means++: The advantages of careful seeding. Technical Report 2006-13, Stanford InfoLab, June 2006.

[9] Meyer F. Topographic distance and watershed lines. Signal Processing 1994;38(1):113-125.

[10] Soille P. Morphological image analysis: principles and applications. Springer Science \& Business Media, 2013.

Address for correspondence:

Name: Roman Jakubicek

Full postal address: Brno University of Technology, Technicka 3082/12, Brno, 616 00, Czech Republic

E-mail address: jakubicek@feec.vutbr.cz 